\title{
Habilidades Sociais e Bem-Estar Subjetivo de Crianças Dotadas e Talentosas
}

\author{
Maria Luiza Pontes de França-Freitas - Universidade Federal do Rio Grande do Norte, Natal, Brasil \\ Almir Del Prette - Universidade Federal de São Carlos, São Carlos, Brasil \\ Zilda A. P. Del Prette - Universidade Federal de São Carlos, São Carlos, Brasil
}

\begin{abstract}
Resumo
O desenvolvimento socioemocional de crianças dotadas e talentosas ainda é objeto de controvérsias na literatura especializada. Este estudo visou avaliar a relação entre habilidades sociais e bem-estar subjetivo de crianças dotadas e talentosas e identificar o poder preditivo da primeira variável sobre a segunda. Participaram 269 crianças, de ambos os sexos, identificadas como dotadas e talentosas, de oito a 12 anos, que responderam a instrumentos padronizados de medida das habilidades sociais e de bem-estar subjetivo. Os resultados mostraram que um repertório elaborado de habilidades sociais está associado à percepção de maior bem-estar subjetivo, podendo inclusive predizê-lo, ou seja, aumentando a probabilidade de relato de afetos positivos, autoconfiança, autoestima e bom-humor, entre outros aspectos.

Palavras-chave: habilidades sociais, bem-estar subjetivo, dotação, talento
\end{abstract}

\begin{abstract}
The socioemotional development of gifted and talented children has been an object of controversy in literature. We aimed (1) to assess the relationship between social skills and subjective well-being in gifted and talented children as well as (2) to identify the predictive power of the first variable on the second. The participants were 269 children identified as gifted and talented with a mean age of 11 years $(\mathrm{SD}=0.91)$. They answered to standardized instruments of social skills and subjective well-being. One found that an elaborate repertoire of social skills is associated with the perception of greater subjective well-being and may also predict this second variable. Therefore an elaborate repertoire of social skills may increase the likelihood of self reported positive affect, self-confidence, self-esteem and good mood, among other aspects.

Keywords: social skills, subject well-being, giftedness, talent
\end{abstract}

Social Skills and Subject Well-Being of Gifted and Talented Children

Resumen

Habilidades Sociales y el Bienestar Subjetivo de Niños Dotados y Talentosos

El desarrollo socioemocional de los niños dotados y talentosos sigue siendo objeto de controversia en la literatura especializada. Este estudio tuvo como objetivo evaluar la relación entre las habilidades sociales y el bienestar subjetivo de esos niños e identificar el poder predictivo de la primera variable sobre la segunda. Participaron 269 niños de ambos sexos identificados como dotados y talentosos con edades entre 8 y 12 años (DT=0,91). Los niños respondieron instrumentos de medición estandarizados de habilidades sociales y de bienestar subjetivo. Los resultados indicaron que un elaborado repertorio de habilidades sociales se encuentran asociadas a la percepción de un mayor bienestar subjetivo, e incluso pueden predecirlo, o sea aumentando cada vez más la probabilidad de reportar las emociones afectivas, confianza en sí mismo, autoestima y buen humor, entre otros aspectos Palabras clave: habilidades sociales, bienestar subjetivo, dotación, talento

Ao considerar os aspectos socioemocionais da vida dos indivíduos dotados e talentosos, uma análise da literatura da área indica que, não obstante sua importância, esses aspectos vêm recebendo menor atenção do que outros, como as características cognitivas e necessidades educacionais desses indivíduos (Alencar, 2007; Fleith, 2007; Rech \& Freitas, 2005), criatividade, sucesso/insucesso, identificação e desenvolvimento do talento (Dai, Swanson, \& Cheng, 2011).

O bem-estar subjetivo é uma das variáveis pouco estudadas na área da dotação e talento. Diener, Lucas e Oishi (2005) definem essa variável como a avaliação cognitiva e afetiva que a pessoa faz sobre a própria vida. De acordo com Diener e Lucas (2000), pode-se entender que o bem-estar apresenta três componentes: Satisfação com a Vida, Afeto Positivo e Afeto Negativo. Segundo os autores citados, bem-estar

Disponivel em wnw.scielo.br subjetivo é um conceito amplo que inclui vivenciar emoções prazerosas, baixos níveis de afeto negativo e altos níveis de satisfação com a vida em geral e em áreas específicas. Referente ao bem-estar subjetivo infantil, Giacomoni (2002) o define como um constructo que remete:

... à vivência de afetos prazerosos, a um ambiente familiar acolhedor, à oportunidades de lazer, aos vinculos com pares, à satisfação de necessidades básicas materiais, à satisfação de alguns desejos, a um ambiente harmônico sem violência, à possibilidade de estudar e se desenvolver cognitivamente e, por fim, às possibilidades de desenvolvimento que promovam um self com características positivas. (p. 148)

Os afetos positivo e negativo estão relacionados com a situação específica atual e a uma grande variedade 
de prazeres, dores e estados emocionais transitórios (Giacomoni, 2002; Kahneman, Diener, \& Scharwz, 1999). Giacomoni (2002) e Giacomoni e Hutz (2008) afirmam que uma avaliação cognitiva de bem-estar é um julgamento avaliativo consciente da pessoa sobre a sua vida como um todo, ou um julgamento sobre aspectos específicos, como o lazer, a família ou a escola.

A satisfação com áreas específicas também contribui para o bem-estar subjetivo de forma mais geral, mas a recíproca pode não ocorrer. Apesar de estar satisfeito com a sua vida em geral, o indivíduo pode não estar satisfeito com algum ou alguns aspectos específicos (Diener, Scollon, \& Lucas, 2003). Em seu estudo de construção e de validação concorrente das escalas para medida do bem-estar subjetivo infantil, Giacomoni (2002) propõe os seguintes indicadores para a avaliação do bem-estar: (a) Self, composto por características positivas (autoestima, bom-humor, capacidade de relacionar-se, capacidade de demonstrar afeto e outras) que descrevem o self como positivo; (b) Self comparado, que seria o agrupamento de itens para avaliações comparativas com os pares referentes ao lazer, à amizade e à satisfação de desejos e afetos; (c) Não violência, conjunto de características associadas a comportamentos agressivos; (d) Família, referindo-se ao ambiente familiar, no quanto pode ser saudável, harmônico, afetivo, de relacionamentos satisfatórios, além de indicações de satisfação quanto à diversão; (e) Amizade, que se refere aos relacionamentos com pares, nível de satisfação desses relacionamentos e algumas indicações ao lazer, diversão e apoio; (f) Escola, importância da escola, qualidade do ambiente escolar, relacionamentos interpessoais nesse espaço e nível de satisfação com relação a esse ambiente.

Entre os indicadores de satisfação de vida, alguns pesquisadores (Ash \& Huebner, 1998; Aspesi, 2007; Diener, Scollon, \& Lucas, 2003; Garces-Bacsal, 2010; Giacomoni, 2002; Huebner, 1991) apontam, como mais significativos, aqueles voltados para o relacionamento interpessoal, como, por exemplo, a satisfação com a família. Garces-Bacsal (2010) enfatiza que os relacionamentos familiares são os principais eventos positivos que geram satisfação no relato de crianças dotadas e talentosas.

Considerando a importância das relações interpessoais, pode-se levantar a hipótese de que o bem-estar esteja relacionado a características socioemocionais das pessoas e, em particular, às suas habilidades sociais. Convergindo com essa hipótese, Neihart (1999) inclui, como indicador de bem-estar, o uso de estratégias para lidar com sentimentos diversos e negociar relações sociais. Efetivamente, o bem-estar da criança pode ser ampliado com a melhoria de seus relacionamentos em diferentes contextos, o que depende, conforme Del Prette e Del Prette (2005), de um bom repertório de habilidades sociais. Cabe destacar que Argyle (1999) também já situava as habilidades sociais entre as variáveis de maior impacto positivo sobre o bem-estar subjetivo e a felicidade.

O termo habilidades sociais se aplica, segundo Del Prette e Del Prette (2001, 2008), às diferentes classes de comportamentos sociais do repertório de um indivíduo, que são requeridas para a qualidade e efetividade das interações que ele estabelece com as demais pessoas. Em outras palavras, as habilidades sociais são componentes indispensáveis da competência social (Del Prette \& Del Prette, 2011).

Existem habilidades sociais específicas e relevantes na infância, como, por exemplo, as que são avaliadas pelo Social Skills Rating System (Gresham \& Elliott, 1990, instrumento adaptado por Bandeira, Del Prette, Del Prette, \& Magalhães, 2009): Responsabilidade, Empatia, Assertividade, Autocontrole, Evitação de Problema e Expressão de Sentimento Positivo. Em vários estudos já conduzidos no Brasil, o repertório social de crianças foi caracterizado com esse instrumento e essas classes (Cia \& Barham, 2009; Del Prette \& Del Prette, 2011; Ferreira, Del Prette, \& Lopes, 2009; Freitas, 2011; Junqueira, 2011).

No instrumento adaptado para o Brasil (Bandeira et al., 2009), a categoria Responsabilidade reúne itens que indicam o compromisso da criança com as tarefas e com as pessoas no ambiente escolar, como, por exemplo: prestar atenção quando o professor está ensinando, seguir suas instruções, fazer as próprias tarefas no tempo estabelecido e usar adequadamente o tempo livre. Na classe Empatia, definida como "a capacidade de compreender e sentir o que alguém sente em uma situação de demanda afetiva, comunicando-lhe adequadamente tal compreensão e sentimento" (Del Prette \& Del Prette, 2001, p. 86), encontram-se comportamentos como: entender quando os colegas estão zangados, aborrecidos ou tristes, ouvir os problemas dos amigos. A Assertividade é definida por Del Prette e Del Prette (2005, p. 175) como "uma classe de habilidades sociais de enfrentamento em situações que envolvem risco de reação indesejável do interlocutor, com controle da ansiedade e expressão apropriada de sentimentos, desejos e opiniões". São exemplos de comportamentos dessa classe: concordar ou discordar 
de opiniões, negociar interesses conflitantes, defender os próprios direitos.

A categoria denominada Autocontrole reúne comportamentos que demonstram domínio sobre as próprias reações emocionais em situações conflituosas com os pais, de pedir e esperar permissão para usar objetos de outrem, de ouvir aqueles que estão falando, de controlar raiva quando zangado(a) (Bandeira et al., 2009). A classe Evitação de problema agrupou comportamentos que demonstram domínio sobre as próprias reações emocionais, como, por exemplo: ignorar colegas fazendo brincadeiras e provocações, discordar de adultos sem briga (Bandeira et al., 2009). Del Prette e Del Prette (2005) destacam que as habilidades de evitação ou solução de problemas interpessoais estão articuladas às demais, complementando as assertivas e empáticas quando o objetivo é garantir maior satisfação pessoal e manutenção da qualidade da relação. A Expressão de sentimento positivo envolve aprovação aos comportamentos dos demais e exteriorização de sentimentos positivos, por exemplo: elogiar e cumprimentar amigos, dizer coisas boas para os outros quando eles fazem alguma coisa bem feita.

Além dessas classes de habilidades sociais, outros conceitos são importantes no campo teórico-prático dessa área, como, por exemplo, automonitoria, considerada pré-requisito para quaisquer outras habilidades sociais (Del Prette \& Del Prette, 2001) e definida como "uma habilidade metacognitiva e afetivo-comportamental pela qual a pessoa observa, descreve, interpreta e regula seus pensamentos, sentimentos e comportamentos em situações sociais" (p. 62). Em uma análise mais recente, Dias e Del Prette (2011) apresentam uma proposta de caracterização de indicadores comportamentais associados ao constructo automonitoria e a seus componentes de autoconhecimento, autorregulação, auto-observação, autocontrole entre outros.

Essas características e outras do campo das habilidades sociais têm sido relacionadas à melhor qualidade de vida, por contribuírem para relações interpessoais mais gratificantes, maior realização pessoal, sucesso profissional, além de melhor saúde física e mental (Bandeira et al., 2009; Lehman \& Erdwins, 2004). As relações interpessoais podem ser melhoradas a partir de habilidades positivas que capacitam os sujeitos nas interações, o que está relacionado com um maior bem-estar subjetivo (Diener \& Fujita, 1995; McCrae \& Costa, 1991). Cia, Pamplin e Del Prette (2006) ressaltam que crianças com características interpessoais positivas (autoestima, autoconceito acadêmico ou não acadêmico, competência social e habilidades específicas de empatia e resolução de problemas) têm maior probabilidade de uma trajetória desenvolvimental satisfatória. A ausência ou o déficit de habilidades sociais são vistos como fatores de risco para problemas comportamentais ou emocionais, entre outros desajustes psicossociais (Cia, Pamplin, \& Del Prette, 2006; Del Prette \& Del Prette, 2005; Feitosa, 2013; Medeiros \& Loureiro, 2004).

A qualidade da relação com os pares e a competência social constituem fatores frequentemente avaliados para o ajustamento psicológico da criança (Neihart, 1999). Não é surpresa, então, que muitos pesquisadores (Bain et al., 2006; Cross, Coleman, \& Stewart, 1995; Galloway \& Porath, 1997; Garland \& Zigler, 1999; Norman, Ramsay, Roberts, \& Martray, 2000; Swiatek, 1995) tentem compreender o ajustamento de crianças dotadas e talentosas pela medida de seu status sociométrico, habilidades de coping social, competência social e habilidades sociais. Pesquisas empíricas têm indicado que as crianças dotadas e talentosas constituem um grupo distinto quando se trata de competência social (Galloway \& Porath, 1997; McCallister, Nash, \& Meckstroth, 1996). Bain e Bell (2004) e França-Freitas, Del Prette e Del Prette (2014) destacam que características comuns em crianças populares, incluindo repertório elaborado de habilidades sociais, poucos problemas de comportamento, habilidades de liderança, acentuado sucesso acadêmico e autoestima elevada, são frequentemente notáveis em crianças dotadas e talentosas. Essas características, afirmam as autoras, podem certamente ter influências positivas na relação e aceitação dos pares. Segundo Hallahan e Kauffman (2003), muitos estudantes dotados ou talentosos são autoconscientes, autoconfiantes e socialmente competentes, variáveis essas relacionadas ao bem-estar.

Lehman e Erdwins (2004) realizaram um estudo com 16 crianças dotadas, analisando algumas variáveis, como ajustamento social e emocional, por meio de testes e identificaram que elas possuíam habilidades sociais que as ajudavam em suas relações interpessoais. O objetivo do estudo dos autores mencionados foi comparar um grupo de crianças dotadas intelectualmente com um grupo de crianças com inteligência média (medidas de quociente de inteligência) em duas medidas de personalidade que fornecem uma variedade de aspectos relevantes sobre o ajustamento social e emocional.

Em nosso país, não obstante alguns estudos tratarem de aspectos socioemocionais de crianças dotadas, verifica-se o predomínio de publicações sobre suas 
características cognitivas e necessidades educacionais, com poucas investigações empíricas, especificamente quantitativas, que deem suporte a eventuais explicações sobre a relação entre habilidades sociais e bem-estar subjetivo de crianças dotadas e talentosas. Conforme Alencar (2007), observa-se predominância de estudos de caso e análises qualitativas, de natureza clínica, com amostras reduzidas e oriundas, muitas vezes, de uma única escola ou programa.

Dado o exposto, o objetivo deste estudo foi investigar uma possível relação entre o repertório de habilidades sociais, em termos de suas diferentes classes, e indicadores de bem-estar subjetivo de crianças dotadas e talentosas. Adicionalmente, buscou-se identificar o poder preditivo das habilidades sociais na explicação do bem-estar subjetivo dessas crianças.

\section{Método}

\section{Participantes}

A amostra foi composta por 269 crianças identificadas como dotadas e talentosas, na faixa-etária entre oito e 12 anos $(M=11$ anos, $D P=0,911)$, a maioria do sexo feminino $(53,2 \%)$. O critério para seleção dos dotados e talentosos foi que a criança, a partir de indicadores de avaliação, demonstrasse dotação em pelo menos um dos domínios da capacidade humana (inteligência geral, criatividade, capacidade socioafetiva e capacidade sensoriomotora). Foram excluídos os indivíduos dotados e talentosos que não frequentavam centros especializados ou que não foram submetidos ao processo padrão de identificação de dotação e talento desses locais. Os participantes da amostra frequentavam escolas regulares e dois centros que possuíam um programa educacional para estudantes dotados e talentosos, integrados aos sistemas de ensino: CEDET (Centro para Desenvolvimento do Potencial e Talento) e DECOLAR (Centro de Desenvolvimento do Talento). Com duração de dois a três anos, o processo de identificação de crianças dotadas é realizado com a participação de três observadores diferentes: dois professores de escola regular de anos diferentes cursados pelos alunos, orientados pelos profissionais do centro e treinados para utilização da lista de indicadores comportamentais de sinalização de dotação e talento (Guenther, 2011); e um facilitador (profissional treinado) dos centros que realiza observação assistida na qual são propocionadas às crianças oportunidades de expressão da capacidade superior, que não foi ou não pode ser observada em uma sala de aula comum.

\section{Instrumentos}

Os participantes responderam a quatro instrumentos de autorrelato: um questionário de caracterização da amostra, uma escala de habilidades sociais e duas escalas de bem-estar subjetivo. A escala de habilidades sociais foi o Sistema de Avaliação de Habilidades Sociais (Social Skills Rating System ou SSRS), produzida originalmente nos EUA (Gresham \& Elliott, 1990) e validada para a amostra brasileira (SSRS-BR, Bandeira, et al., 2009). O SSRS-BR apresenta itens de situações e reações que permitem avaliar habilidades sociais, comportamentos problemáticos e competência acadêmica. É apresentada sob três versões: a Versão P para professores (30 itens); a Versão M para os pais (37 itens); e a Versão C para a própria criança (27 itens). Neste estudo, foi utilizada a Versão C, de autoavaliação, que avalia o repertório de habilidades sociais com base em uma escala de frequência $(0=$ nunca; $1=$ algumas vezes; 2 = muito frequente), composta por seis subescalas, com eigenvalues entre 1,16 e 4,09, que explicam 41, $65 \%$ da variância dos dados: responsabilidade, empatia, assertividade, autocontrole, evitação de problemas e expressão de sentimento positivo. O SSRS-BR apresenta propriedades psicométricas satisfatórias e foi recentemente recomendado pelo SATEPSI do Conselho Federal de Psicologia (http://satepsi.cfp.org.br/, recuperado em 08, dezembro, 2014). O SSRS-BR possui satisfatória consistência interna, aferida pelo alfa de Cronbach nas escalas de habilidades sociais (estudante $=0,78$; pais $=0,86$; professores $=0,94)$; de comportamentos problemáticos (pais $=0,83$; professores $=0,91$ ) e de competência acadêmica (alfa $=0,98)$.

Para avaliação do Bem-estar subjetivo foram utilizadas duas escalas, uma referente à Satisfação com a Vida e outra ao Afeto Positivo e Negativo. A Escala Multidimensional de Satisfação de Vida para Crianças (EMSVC), elaborada por Giacomoni e Hutz (2008), com 50 itens, do tipo Likert, cada um permitindo resposta que varia de 1 (nem um pouco) a 5 (muitíssimo). Os itens se reúnem em seis fatores, que explicam $46,5 \%$ da variância total e que são denominados de: Self (exemplo de item: Eu sou alegre), Self Comparado (exemplo de item: Meus amigos são mais alegres do que eu), Não Violência (exemplo de item invertido: Brigo muito com meus amigos), Família (exemplo de item: Meus pais são carinhosos comigo), Amizade (exemplo de item: Estou satisfeito com os amigos que tenho) e Escola (exemplo de item: Eu gosto de ir à escola). Os autores encontraram consistências internas adequadas para cada subescalas $(0,82$ a 0,86$)$, assim como para a escala 
total $(0,93)$. A validade concorrente foi confirmada por correlações médias com medidas critério.

A Escala de Afeto Positivo e Negativo para Crianças, elaborada por Giacomoni e Hutz (2006), é composta por 30 termos nos quais o respondente pontua diferentes sentimentos e emoções (15 itens de afeto positivo e 15 de afeto negativo). Exemplos de alguns dos termos que compõem a escala utilizada são: alegre, impaciente, feliz, envergonhado, amoroso, irritado, triste. A escala também é tipo Likert e varia de 1 (nem um pouco) a 5 (muitíssimo). Os coeficientes alfa de Cronbach das subescalas de Afeto Positivo $(0,88)$ e de Afeto Negativo $(0,84)$ apontam evidências de confiabilidade e a escala apresenta boas evidências de validação concorrente. A partir de análises fatoriais os autores confirmaram a estrutura da escala por meio da solução de dois fatores.

\section{Procedimento}

O presente estudo foi aprovado pelo Comitê de Ética em Pesquisa da Universidade Federal de São Carlos. Cumpridas as exigências éticas, assegurado o anonimato e verificada a compreensão sobre a pesquisa pela escola, pais e crianças, iniciou-se a coleta de dados que ocorreu nos centros e nas escolas regulares das crianças.

Foi discutido com as coordenadoras e facilitadoras a melhor forma de coletar os dados das crianças matriculadas nos centros, de modo a não atrapalhar a rotina das atividades realizadas. Em seguida, a aplicação dos instrumentos foi planejada de modo a evitar o efeito de exposição de informação e durante esse processo foi enfatizado o caráter voluntário da participação e dadas instruções às crianças quanto ao preenchimento e resposta aos questionários, que durava em média 30 minutos. As crianças também foram informadas de que as facilitadoras do centro, as professoras da escola regular e os pais não teriam acesso ao conteúdo do questionário preenchido por elas.

\section{Análise dos Dados}

Para a obtenção dos resultados, os dados foram organizados em planilhas do pacote estatístico Predictive Analytics Software Statistics (PASW, Statistics Base for $W$ indows, versão 18.0). Foram efetuadas análises de correlação $r$ de Pearson (com teste de significância two-tailed) entre os indicadores de habilidades sociais e bem-estar subjetivo. Para estimar o poder preditivo das habilidades sociais em relação ao bem-estar subjetivo de crianças dotadas e talentosas, foram realizadas quatro análises de regressão linear múltipla, adotando-se o método stepwise, para verificar o quanto as habilidades sociais podem contribuir para explicar a variância dos escores de Bem-estar subjetivo e seus indicadores (Satisfação com a Vida, Afeto Positivo e Afeto Negativo).

\section{Resultados}

A análise das correlações mostrou pontuações moderadas entre os construtos Habilidades sociais e Bem-estar subjetivo. Estas podem ser visualizadas na Tabela 1.

O escore total de Habilidades sociais apresentou correlação positiva moderada com o escore total de Bem-estar subjetivo. O mesmo ocorreu para todos os indicadores de bem-estar subjetivo em correlação com o escore total de Habilidades sociais, sendo maior para Satisfação com a Vida. Considerando as subescalas de habilidades sociais, as correlações foram mais fortes com o escore total Bem-estar subjetivo com as habilidades de Responsabilidade, seguida por Autocontrole e Assertividade. Surpreendentemente, a única classe de habilidade social que não apresentou correlação com o escore total de Bem-estar subjetivo foi Empatia.

Considerando separadamente os indicadores de bem-estar subjetivo, verificou-se correlação significativa com as habilidades sociais de Responsabilidade, Assertividade, Autocontrole e Evitação de Problemas, mas não com as subescalas de Empatia e de Expressão de Sentimento Positivo. Esta última classe apresentou correlação negativa baixa, mas não significativa apenas com o indicador Afeto Negativo, enquanto Empatia foi a subescala de Habilidade Social que apresentou a menor quantidade de correlações significativas com os indicadores de bem-estar subjetivo, sendo essas correlações fracas ainda que na direção esperada, por exemplo, escore total de Satisfação com a Vida e subescala de Amizade. Outras correlações encontradas merecem destaque, como no caso das habilidades da subescala Responsabilidade, que apresentou correlações moderadas com os indicadores Satisfação com Escola, seguido pelo escore total de Satisfação com a Vida.

Ao considerar outras correlações com maior força, apresentadas na Tabela 1, destacam-se as seguintes: (a) o indicador de bem-estar subjetivo Não Violência apresentou correlação moderada com as habilidades sociais de Autocontrole e Responsabilidade; (b) o indicador de bem-estar subjetivo Satisfação com Amizade apresentou maior correlação positiva com a habilidade de Assertividade seguida por Expressão de Sentimento 
Tabela 1

Coeficientes de Correlação para Subescalas de Habilidades Sociais e Indicadores de Bem-Estar Subjetivo

\begin{tabular}{lccccccc}
\hline \multirow{2}{*}{$\begin{array}{l}\text { Bem-estar } \\
\text { subjetivo }\end{array}$} & $\begin{array}{c}\text { Escore } \\
\text { Total }\end{array}$ & Respons. & Empat. & Assert. & Autocont. & Evitação & Expres. \\
\hline BES & $0,56^{* * *}$ & $0,47^{* * *}$ & 0,08 & $0,42^{* * *}$ & $0,43^{* * *}$ & $0,30^{* * *}$ & $0,37^{* * *}$ \\
Af.negat. & $-0,30^{* * *}$ & $-0,31^{* * *}$ & 0,08 & $-0,23^{* * *}$ & $-0,31^{* * *}$ & $-0,17^{* *}$ & $-0,06$ \\
Af.posit. & $0,44^{* * *}$ & $0,31^{* * *}$ & 0,08 & $0,38^{* * *}$ & $0,29^{* * *}$ & $0,21^{* * *}$ & $0,40^{* * *}$ \\
S. global & $0,57^{* * *}$ & $0,46^{* * *}$ & $0,15^{*}$ & $0,40^{* * *}$ & $0,40^{* * *}$ & $0,31^{* * *}$ & $0,40^{* * *}$ \\
Sself & $0,50^{* * *}$ & $0,38^{* * *}$ & $0,14^{* *}$ & $0,35^{* * *}$ & $0,35^{* * *}$ & $0,26^{* * *}$ & $0,37^{* * *}$ \\
Sselfcomp. & $0,25^{* * *}$ & $0,21^{* * *}$ & $0,12^{*}$ & $0,13^{*}$ & $0,13^{* *}$ & $0,20^{* * *}$ & $0,16^{*}$ \\
Snãoviol. & $0,43^{* * *}$ & $0,40^{* * *}$ & 0,04 & $0,29^{* * *}$ & $0,44^{* * *}$ & $0,16^{* *}$ & $0,16^{*}$ \\
Sfamília & $0,40^{* * *}$ & $0,32^{* * *}$ & 0,03 & $0,27^{* * *}$ & $0,35^{* * *}$ & $0,19^{* *}$ & $0,34^{* * *}$ \\
Samizade & $0,44^{* * *}$ & $0,28^{* * *}$ & $0,13^{*}$ & $0,37 * * *$ & $0,25^{* * *}$ & $0,29^{* * *}$ & $0,35^{* * *}$ \\
Sescola & $0,51^{* * *}$ & $0,52^{* * *}$ & 0,12 & $0,34^{* * *}$ & $0,35^{* * *}$ & $0,18^{* *}$ & $0,31^{* * *}$ \\
\hline
\end{tabular}

Nota. Significativamente estatístico para $* p<0,05 ; * * p<0,01 ; * * *<0,001$ (two-tailed).

BES = Bem-estar Subjetivo; Af.negat. = Afeto negativo; Af.Posit = Afeto negativo; S.global = Satisfação com a vida global; Sself = Self; Sselfcomp. $=$ Self comparado; Snãoviol. $=$ Não violência; Sfamília $=$ Satisfaçaõ com a família; Samizade $=$ Satisfação com amizade; Sescola $=$ Satisfação com a escola; Respns = Responsabilidade; Empat = Empatia; Assert = Assertividade; Autocont = Autocontrole; Evitação = Evitação de Problema; Expres $=$ Expressão de Sentimento Positivo.

Positivo; (c) o índice mais alto de correlação do escore total de Satisfação com a Vida foi verificado com a habilidade de Responsabilidade; (d) o índice de correlação do indicador Afeto Positivo foi maior com a categoria de habilidade social de Expressão de Sentimento Positivo.

$\mathrm{O}$ indicador de bem-estar subjetivo Self Comparado e a classe Empatia foram as variáveis que apresentaram maior número de correlações fracas em sua associação com as demais variáveis. Além disso, como esperado, o único indicador de bem-estar subjetivo que apresentou correlações negativas com o escore total e com as subescalas de habilidades sociais foi o de Afeto Negativo. Esse indicador apresentou maior força na correlação com as subescalas Responsabilidade e Autocontrole, ainda que também fracas. Destaca-se que a maioria das correlações obtidas na análise apresentou nível de significância menor que 0,001.

Os resultados da análise de regressão são apresentados na Tabela 2. Os modelos foram testados para cada uma das principais variáveis (critério), tomando-se as demais como possíveis antecedentes.

O primeiro modelo, que teve como variável critério o escore total de Bem-Estar Subjetivo, apresentou, como variáveis antecedentes, Responsabilidade, Assertividade, Autocontrole e Expressão de Sentimento Positivo. Os resultados indicaram que essas subescalas de Habilidades Sociais predizem o Bem-Estar Subjetivo, com um percentual de explicação de $33 \%$, o que significa que quanto mais elaborado o repertório dessas classes de Habilidades Sociais, maior o nível de Bem-Estar Subjetivo relatado.

O segundo modelo teve como variável critério a Satisfação com a Vida e apresentou, como preditoras, as subescalas Responsabilidade, Expressão de Sentimento Positivo, Evitação de Problema e Autocontrole. A associação entre as variáveis critério e seus antecedentes é moderadamente forte $\left(R_{\text {múltiplo }}\right.$ $=0,60)$. Juntas, essas classes de habilidades sociais foram responsáveis por $34 \%$ da variância no indicador Satisfação com a Vida $\left(R_{\text {ajustado }}^{2}=0,34\right)$. Ao analisar a Tabela 2, verifica-se que os coeficientes de regressão padronizados indicam que Responsabilidade foi mais forte que Expressão de Sentimento Positivo, Evitação de Problema e Autocontrole, na explicação da variável Satisfação com a Vida.

O terceiro modelo, que teve como variável critério Afeto Positivo apresentou, como variáveis 
Tabela 2

Subescalas de Habilidades Sociais Preditoras do Bem-Estar Subjetivo e seus Indicadores

\begin{tabular}{|c|c|c|c|c|}
\hline \multirow{3}{*}{ Preditores } & \multicolumn{4}{|c|}{ Bem-Estar Subjetivo } \\
\hline & Modelo 1 & Modelo 2 & Modelo 3 & Modelo 4 \\
\hline & Beta & Beta & Beta & Beta \\
\hline Responsabilidade & $0,48^{* * *}$ & $0,36^{* * *}$ & $0,27 * * *$ & $0,25^{* * *}$ \\
\hline Assertividade & ------ & $0,29 * * *$ & $0,26^{* * *}$ & $0,20 * * *$ \\
\hline Autocontrole & ------ & ------ & $0,20 * * *$ & $0,19 * * *$ \\
\hline Expressão Sent. & ------ & ------ & ------ & $0,16^{* *}$ \\
\hline F & 74,38 & 55,08 & 42,07 & 34,46 \\
\hline \multirow[t]{2}{*}{$R_{\text {ajustado }}^{2}$} & .21 & .28 & .31 & .33 \\
\hline & \multicolumn{4}{|c|}{ Satisfação com a Vida } \\
\hline \multirow[t]{2}{*}{ Preditores } & Modelo 1 & Modelo 2 & Modelo 3 & Modelo 4 \\
\hline & Beta & Beta & Beta & Beta \\
\hline Responsabilidade & $0,46 * * *$ & $0,38^{* * *}$ & $0,36^{* * *}$ & $0,27 * * *$ \\
\hline Expressão Sent. & ------ & $0,30 * * *$ & $0,25^{* * *}$ & $0,24 * * *$ \\
\hline Evitação Problema & ------ & ------ & $0,19 * * *$ & $0,18^{* * *}$ \\
\hline Autocontrole & ------ & ------ & ------ & $0,17 * *$ \\
\hline$F$ & 72,04 & 54,27 & 42,40 & 34,83 \\
\hline \multirow[t]{2}{*}{$R_{\text {ajustado }}^{2}$} & .21 & .28 & .32 & .34 \\
\hline & \multicolumn{4}{|c|}{ AFETO POSITIVO } \\
\hline \multirow[t]{2}{*}{ Preditores } & Modelo 1 & Modelo 2 & Modelo 3 & ------ \\
\hline & Beta & Beta & Beta & ------ \\
\hline Expressão & $0,40^{* * *}$ & $0,29 * * *$ & $0,26 * * *$ & ------ \\
\hline Assertividade & ------ & $0,26^{* * *}$ & $0,22 * * *$ & ------ \\
\hline Responsabilidade & ------ & ------ & $0,16^{* *}$ & ------ \\
\hline$F$ & 50,12 & 36,21 & 27,22 & ------ \\
\hline \multirow[t]{2}{*}{$R_{\text {ajustado }}^{2}$} & .16 & .21 & .23 & ------ \\
\hline & \multicolumn{4}{|c|}{ AFETO NEGATIVO } \\
\hline \multirow[t]{2}{*}{ Preditores } & Modelo 1 & Modelo 2 & Modelo 3 & Modelo 4 \\
\hline & Beta & Beta & Beta & Beta \\
\hline Autocontrole & $-0,32 * * *$ & $-0,21 * *$ & $-0,23 * * *$ & $-0,22 * * *$ \\
\hline Responsabilidade & ------ & $-0,20 * *$ & $-0,21 * *$ & - 0,19** \\
\hline Empatia & ------ & ------ & $0,15^{*}$ & $0,18 * *$ \\
\hline Evitação & ------ & ------ & ------ & $-0,15^{*}$ \\
\hline F & 29,34 & 19,61 & 15,50 & 13,44 \\
\hline$R_{\text {ajustado }}^{2}$ & .10 & .12 & .14 & .16 \\
\hline
\end{tabular}

Nota. Significativamente estatístico para $* p<0,05$; ** $p<0,01$; *** $p<0,001$. 
antecedentes, as habilidades de Expressão de Sentimento Positivo, Assertividade e Responsabilidade. Como se vê na Tabela 2, os resultados indicaram que o conjunto das variáveis antecedentes explicou $23 \%$ $\left(R_{\text {ajustado }}^{2}=0,23\right)$ da variância total $\left(R_{\text {múltiplo }}=0,49\right)$, na variável critério, sugerindo que, quanto maior a pontuação nessas subescalas maior o nível de Afeto Positivo relatado pelas crianças dotadas.

No quarto modelo, o indicador Afeto Negativo foi negativamente predito pelas subescalas Autocontrole, Responsabilidade e Evitação de Problemas e positivamente pela classe Empatia. Os resultados, apresentados na Tabela 2, indicaram que essas subescalas de habilidades sociais predizem esse indicador, com um percentual de explicação de 16\%. Esses resultados sugerem que quanto maior a pontuação relatada pelas crianças dotadas nas subescalas Autocontrole, Responsabilidade e Evitação de Problema, menor a pontuação no indicador Afeto Negativo e, ao contrário, quanto maior a pontuação das crianças em Empatia maior a probabilidade de relato de Afeto Negativo.

Nos dois primeiros modelos, Responsabilidade foi a classe de habilidade social que apresentou coeficiente de regressão mais forte na explicação do Bem-Estar Subjetivo e do escore geral Satisfação com a Vida. Como puderam ser verificadas na Tabela 2, as habilidades sociais da classe Responsabilidade foram preditoras relevantes nos quatro modelos explicativos.

\section{Discussão}

Com base nos resultados obtidos, foi possível verificar que, quanto mais desenvolvido o repertório de habilidades sociais maior o nível de bem-estar subjetivo relatado pelas crianças dotadas e talentosas. Apesar de não serem encontrados estudos relacionando as variáveis mencionadas para essa população específica, vários estudos (Argyle, 1999; Bandeira et al., 2009; Diener \& Fujita, 1995; Lehman \& Erdwins, 2004; McCrae \& Costa, 1991) têm indicado que as características sociais do indivíduo, dentre elas as habilidades sociais, têm sido relacionadas ao bem-estar subjetivo, isso porque possibilitam relações interpessoais mais gratificantes, maior realização pessoal, sucesso profissional, além de melhor saúde física e mental e, consequentemente, melhor qualidade de vida.

$\mathrm{O}$ fato de ter sido evidenciado que quanto mais elaborado o repertório de habilidades sociais maior é o nível de bem-estar subjetivo em crianças dotadas e talentosas pode indicar que estas possivelmente têm vivenciado afetos prazerosos, provavelmente em um ambiente familiar acolhedor, vínculos fortalecidos com os pares, possibilidade de desenvolver seu potencial, bem como podem receber mais reforços positivos em consequência do seu desempenho social. Chamam a atenção, também, algumas particularidades dos resultados no que diz respeito às relações obtidas entre subescalas específicas de Habilidades Sociais e os indicadores de Bem-Estar Subjetivo.

Destaca-se que foram encontradas correlações moderadas positivas entre as habilidades de Expressão de Sentimento Positivo e dois indicadores de bem-estar subjetivo, Satisfação com a Vida e Afeto Positivo. Além disso, esses dois indicadores e Bem-Estar Subjetivo Geral foram preditos por essa classe de habilidade social. É possível afirmar, com base nos dados obtidos, que as crianças dotadas e talentosas, que exteriorizam sentimentos positivos e expressam aprovação aos comportamentos dos demais, experienciam maior frequência de emoções positivas, como alegria, contentamento, felicidade, amor, bem como satisfação com a vida. Esses dados são semelhantes aos do estudo de Lehman e Erdwins (2004) no qual crianças dotadas demonstraram habilidades sociais que facilitavam suas relações interpessoais e, desse modo, contribuíam para relações mais satisfatórias e melhor qualidade de vida. Argyle (1999) afirma que expressar sentimentos positivos contribui para desenvolver relações interpessoais mais gratificantes, como, por exemplo, relações afetuosas entre familiares e, consequentemente, um maior bem-estar subjetivo.

Outras correlações que merecem destaque ocorreram entre as habilidades de Expressão de Sentimento Positivo e Satisfação com Amizade, e entre esta e Assertividade. Esse resultado pode indicar que crianças dotadas, que expressam apropriadamente seus sentimentos, desejos e opiniões, mantém a qualidade de suas relações com amigos, obtendo maior satisfação com amizades.

Habilidades de Assertividade envolvem enfrentamento de situações que demandam controle da ansiedade ou outras emoções negativas, bem como expressão apropriada de sentimentos, desejos e opiniões. Verificou-se que as crianças dotadas e talentosas que relataram maior escore de Assertividade relataram mais Afeto Positivo e níveis altos de Bem-Estar Subjetivo. Expressar o que se pensa e o que se sente pode contribuir para solucionar conflitos, diminuindo a probabilidade de afetos negativos e criando condições de melhor equilíbrio emocional. 
Em relação às habilidades de Autocontrole, como esperado, quanto mais demonstravam, por meio do autorrelato, domínio sobre as próprias reações emocionais mais frequentemente se consideravam satisfeitas com a própria vida. Entre as crianças dotadas, as que relataram repertório mais elaborado em habilidades de Autocontrole afirmaram sentir, em menor frequência, emoções negativas, como tristeza, irritação, desânimo, medo, impaciência, perturbação, nervosismo e outras. Somado a essa predição negativa, a correlação moderada dessa classe com o indicador de bem-estar subjetivo Não Violência pode indicar a importância das habilidades de automonitoria, para as quais o autocontrole é uma dimensão central (Del Prette \& Del Prette, 2001) que contribui para a competência social e para o sucesso no enfrentamento de situações que poderiam facilitar respostas agressivas. Aquelas pessoas que aprenderam a se automonitorar são geralmente conscientes de suas emoções (Del Prette \& Del Prette, 2001), o que está associado ao autocontrole e contribui para a competência social. Outros componentes da automonitoria, como a identificação das emoções, em si e nos outros, somada à adequada leitura dos sinais sociais do ambiente, são condições necessárias para a criança decidir pela omissão ou pela expressão adequada de uma emoção (Del Prette \& De Prette, 2005). Nesse sentido, como o Autocontrole implica domínio sobre as próprias reações emocionais (Bandeira et al., 2009), é possível que essa característica tenha contribuído para o autocontrole das crianças de modo a evitarem brigas ou falarem/agirem impulsivamente.

No caso específico das habilidades de Responsabilidade, a correlação mais forte foi com o indicador Satisfação com a Escola. Essa correlação pode ser explicada pelo fato dessa classe de habilidade social se referir a comportamentos que demonstram compromisso da criança com as tarefas e com as pessoas no ambiente escolar. É mais provável que crianças dotadas entendam as instruções dos professores e sejam capazes de realizarem as tarefas solicitadas obtendo acentuado sucesso acadêmico (Bain \& Bell, 2004) e, com isso, maior satisfação com o ambiente escolar. A forte correlação entre os escores de Responsabilidade e o indicador de Não violência pode também indicar o papel protetor dessas habilidades no contexto escolar especialmente as de seguimento de regras e instruções e de uso adequado do tempo livre.

Pode-se destacar que, dentre as subescalas de habilidades sociais, as de Responsabilidade foram as que mais explicaram a variância de todos os demais indicadores analisados (Bem-Estar Subjetivo, Satisfação com a Vida, Afeto Positivo e Afeto Negativo). Possivelmente, essas são habilidades que atendem às exigências do meio ambiente, diminuindo a probabilidade das crianças serem punidas (Afeto Negativo) e aumentando a de serem positivamente reforçadas (Afeto Positivo). Assim, os dados deste estudo permitem reafirmar, conforme a literatura da área, que um repertório elaborado de habilidades sociais pode contribuir para a prevenção de comportamentos agressivos, dificuldades de aprendizagem e problemas psicossociais (Del Prette \& Del Prette, 2005) e tem impacto positivo sobre o bem-estar subjetivo, a felicidade (Argyle, 1999) e, consequentemente, a qualidade de vida.

No que diz respeito à Empatia, um dado intrigante foi sua correlação com Afetos Negativos. Pode-se levantar a hipótese de que esse resultado seja devido aos itens de empatia do SSRS-BR estarem se referindo a condições e sentimentos "negativos" de tristeza, por exemplo, "Eu fico triste pelos outros quando coisas ruins lhe acontecem, Eu ouço meus amigos quando eles falam de problemas deles" e "Eu tento entender como meus amigos se sentem quando estão zangados, aborrecidos ou tristes". A sensibilidade a essas emoções nos outros (ainda que positiva do ponto de vista de um repertório elaborado de empatia) pode ter estimulado a percepção de afeto negativo aumentado e de situações de injustiça para essas crianças (Silverman, 1994). De todo modo, trata-se de uma questão empírica a ser mais bem investigada.

Foi possível verificar neste estudo que as crianças que relataram repertório mais elaborado de Evitação de Problema, também reportaram maior Satisfação com a Vida e menos Afetos Negativos, como tristeza, irritação, desânimo, medo e outros. Esse repertório certamente sugere maior capacidade da criança em lidar com as fontes potenciais de estresse e autocontrole de reações impulsivas (Del Prette \& Del Prette, 2005), o que provavelmente contribui para a satisfação com a vida e a evitação de situações de afeto negativo.

Em resumo, o presente estudo mostrou que o bem-estar subjetivo foi predito pela maioria das subescalas de habilidades sociais, o que permite reafirmar o papel desse repertório para a qualidade de vida, inclusive entre crianças dotadas e talentosas. Entende-se que esses resultados trazem algumas contribuições para a compreensão e, possivelmente, a promoção do bem-estar e qualidade de vida das pessoas dotadas e talentosas. 
Não obstante, essas potenciais contribuições são reconhecidas como algumas limitações do presente estudo. A primeira é o fato de basear-se no autorrelato dos participantes. Apesar de o bem-estar ser um estado subjetivo, na avaliação de habilidades sociais tem sido recomendada uma abordagem multimodal (Del Prette \& Del Prette, 2003), o que implica avaliação com diferentes instrumentos, procedimentos e informantes. Outra limitação é o tamanho da amostra que, embora suficiente para as análises realizadas, restringem a possibilidade de generalização dos dados para indivíduos de outros contextos brasileiros. Pesquisas futuras deveriam realizar avaliação multimodal e garantir a amostragem com crianças de outras regiões do país.

\section{Referências}

Alencar, E. M. L. S. (2007). Características socioemocionais do superdotado: Questões atuais. Psicologia em Estudo, Maringá, 12(2), 371-378.

Argyle, M. (1999). Causes and correlates of happiness. Em D. Kahneman, E. Diener \& N. Schwarz (Eds.), Well-being: The foundations of hedonic psychology (pp. 354-373). New York: Russell Sage Foundation.

Ash, C., \& Huebner, E. S. (1998). Life satisfaction reports of gifted middle-school children. School Psychology Quartely, 13(4), 310-321.

Aspesi, C. C. (2007). A família do aluno com altas habilidades/superdotação. Em D. S. Fleith (Ed.), $A$ construção de práticas educacionais para alunos com altas habilidades/superdotação: $O$ aluno e a familia (pp. 2947). Brasília: MEC/SEESP.

Bain, S. K., \& Bell, S. M. (2004). Social self-concept, social attributions, and peer relationships in fourth, fifth, and sixth graders who are gifted compared to high achievers. Gifted Child Quarterly, 48(3), 168-178.

Bain, S. K., Choate, S. M., \& Bliss, S. L. (2006). Perceptions of developmental, social, and emotional issues in giftedness: Are they realistic? Roeper Review, 29(1), 41-48.

Bandeira, M., Del Prette, Z. A. P., Del Prette, A., \& Magalhães, T. (2009) Validação das escalas de habilidades sociais, comportamentos problemáticos e competência acadêmica (SSRS-BR) para o ensino fundamental. Psicologia: Teoria e Pesquisa, 25(2), 271-282.
Cia, F., \& Barham, E. J. (2009). Repertório de habilidades sociais, problemas de comportamento, autoconceito e desempenho acadêmico de crianças no início da escolarização. Estudos de Psicologia (Campinas), 26(1), 45-55.

Cia, F., Pamplin, R. C. O., \& Del Prette, Z. A. P. (2006). Comunicação e participação pais-filhos: Correlação com habilidades sociais e problemas de comportamento dos filhos. Paidéia, 16(35), 395-406.

Cross, T. L., Coleman, L. J., \& Stewart, R. A. (1995). Psychosocial diversity among gifted adolescents: An exploratory study of two groups. Roeper Review, 17(3), 181-185.

Dai, D. Y., Swanson, J. A., \& Cheng, H. (2011).State of research on giftedness and gifted education: A survey of empirical studies published during 1998 -2010 (april). Gifted Child Quarterly, 55 (2), 126-138.

Del Prette, A., \& Del Prette, Z. A. P. (2001). Psicologia das relaçôes interpessoais: Vivências para o trabalho em grupo ( $6^{\mathrm{a}}$ ed.). Petrópolis: Vozes.

Del Prette, Z. A. P., \& Del Prette, A. (2003). Habilidades sociais e dificuldades de aprendizagem: Teoria e pesquisa sob um enfoque multimodal. Em A. Del Prette \& A. P. Del Prette (Eds.), Habilidades sociais, desenvolvimento e aprendizagem: questões conceituais, avaliação e intervenção (pp. 167-206). Campinas: Alínea.

Del Prette, Z. A. P., \& Del Prette, A. (2005). Psicologia das habilidades sociais na infância: Teoria e prática. Petrópolis: Vozes.

Del Prette, Z. A. P., \& Del Prette, A. (2008). Um sistema de categorias de habilidades sociais educativas. Paidéia, 18(41), 517-530.

Del Prette, Z. A. P., \& Del Prette, A. (2011). Práticas baseadas em evidência e treinamento de habilidades sociais. Em A. Del Prette \& Z. A. P. Del Prette (Eds.), Habilidades sociais: Intervenções efetivas em grupo. (pp. 19-56). São Paulo: Casa do Psicólogo.

Dias, T. P. \& Del Prette, Z. A. P. (2011). Automonitoria: História, definição e importância para o campo das habilidades sociais. Anais do $3^{\circ}$ Seminário Internacional de Habilidades Sociais, Taubaté, São Paulo, p. 10.

Diener, E., \& Lucas, R. E. (2000). Explaining differences in societal levels of happiness: Relative standards, need fulfillment, culture, and evaluation theory. Journal of Personality Assessement, 1(1), 41-78. 
Diener, E., \& Fujita, F. (1995). Resources, personal strivings, and subjective well-being: A nomothetic and idiographic approach. Journal of Personality and Social Psychology, 68(5), 926-935.

Diener, E., Lucas, R. E., \& Oishi, S. (2005). Subjective well-being: The science of happiness and life satisfaction. Em C. R. Snyder \& S. J. Lopez (Eds.), Handbook of Positive Psychology (pp. 63-73). New York: Oxford University Press.

Diener, E., Scollon, C., Lucas, R. (2003). The evolving concept of subjective well-being: The multifaceted nature of happiness. Em P. T. Costa \& I. C. Siegler (Eds.), Advances in cell aging and gerontology (Vol. 15, pp. 187-219). Amsterdam: Elsevier Science \& Technology.

Feitosa, B. F. (2013). Habilidades sociais e sofrimento psíquico. Arquivos Brasileiros de Psicologia, 65 (1), 38-50.

Ferreira, B. C., Del Prette, Z., \& Lopes, D. C. (2009). Habilidades empáticas de crianças videntes e cegas e a possível influência de variáveis sociodemográficas. Interação em Psicologia, 13(1), 49-58.

Fleith, D. S. (2007). Conceitos e práticas na educação de alunos com altas habilidades/superdotação. Revista Brasileira de Educação Especial, 13(3), 479-480.

França-Freitas, M. L. P.; Del Prette, A.; Del Prette, Z. A. P. (2014). Social skills of gifted and talented children. Estudos de Psicologia (UFRN), 19 (4), 288-295.

Freitas, L. C. (2011). Habilidades sociais de crianças com diferentes necessidades educacionais especiais: Comparações múltiplas (Tese de doutorado em Educação Especial). Universidade Federal de São Carlos, São Carlos, SP.

Galloway, B., \& Porath, M. (1997). Parent and teacher views of gifted children's social abilities. Roeper Review, 20(2), 118-121.

Garces-Bacsal, R. M. (2010). Tales gifted children tell: Exploring PTAT responses as pathways to socio-affective concerns. Gifted Child Quarterly, 54(2), 138-151.

Garland, A. F., \& Zigler, E (1999). Emotional and behavioral problems among highly intellectually gifted youth. Roeper Review, 22(1), 41-44.

Giacomoni, C. H. (2002). Bem-Estar subjetivo infantil: Conceito de felicidade e construção de instrumento para avaliação
(Tese de doutorado em Psicologia). Universidade Federal do Rio Grande do Sul, Porto Alegre, RS.

Giacomoni, C. H., \& Hutz, C. S. (2006). Escala de afeto positivo e negativo para crianças: Estudos de construção e validação. Psicologia Escolar e Educacional, 10(2) 235-245.

Giacomoni, C. H., \& Hutz, C. S. (2008). Escala multidimensional de satisfação com a vida para crianças: Estudos de construção e validação. Estudos de Psicologia, 25(1), 23-35.

Gresham, F. M., \& Elliott, S. N. (1990). Social Skills Rating System: Manual. Circle Pines, MN: American Guidance Service.

Guenther, Z. C. (2011). Caminhos para desenvolver potencial e talento. Lavras: Ed. UFLA.

Hallahan, D. P., \& Kauffman, J. M. (2003). Exceptional learners: Introduction to special education. Boston: Allyn and Bacon.

Huebner, E. S. (1991). Initial development of the student's life satisfaction scale. School Psychology International, 12, 229-238.

Junqueira, P. H. (2011). Análise funcional de interações sociais favoráveis e desfavoráveis à inclusão de crianças em escola regular (Tese de doutorado em Psicologia). Universidade Federal de São Carlos, São Carlos, SP, 187p.

Kahneman, D., Diener, E. \& Schwarz, N. (1999). Understanding well-being: Scientific perspectives on enjoyment and suffering. New York: Russell-Sage.

Lehman, E. B., \& Erdwins, C. J. (2004). The social and emotional adjustment of young intellectually gifted children. Em S. Moon (Ed.), Social/emotional issues, underachievement and counseling of gifted and talented students (pp.1-8).Thousand Oaks: Corwin.

McCallister, C., Nash, W. R., \& Meckstroth, E. (1996). The social competence of gifted children: Experiments and experience. Roeper Review, 18(4), 273-276.

McCrae, R. R., \& Costa, P. T. (1991). Adding liebe und arbeit: The full five-factor model and wellbeing. Personality and Social Psychology Bulletin, 17(2), 227-232.

Medeiros, P. C. \& Loureiro, S. R. (2004). Observação clínica do comportamento de crianças com queixa de dificuldade de aprendizagem. Em E. M. Marturano, M. B. M. Linhares \& S. R. Loureiro (Orgs.), Vulnerabilidade e proteção: Indicadores na trajetória 
de desenvolvimento escolar (p. 107-122). São Paulo: Casa do Psicólogo.

Neihart, M. (1999). The impact of giftedness on psychological well-being: What does the empirical literature say? Roeper Review, 22(1), 10-17.

Norman, A. D., Ramsay, S. G., Roberts, J. L., \& Martray, C. R. (2000).Effects of social setting, self-concept, and relative age on the social status of moderately and highly gifted students. Roeper Review, 23(1), 34-39.

Rech, A. J. D., \& Freitas, S. N. (2005). Uma análise dos mitos que envolvem os alunos com altas habilidades: A realidade de uma escola de Santa Maria/RS. Revista Brasileira de Educação Especial, 11(2), 295-314.
Sistema de Avaliação de Testes Psicológicos (2014). SSRS-BR. Sistema de Avaliação de Testes Psicológicos (SATEPSI). Recuperado de http://satepsi. cfp.org.br/lista'Teste.cfm?status $=$

Silverman, L. K. (1994). The moral sensitivity of gifted children and the evolution of society. Roeper Review, 17( 2 ), 110-116.

Swiatek, M. A. (1995). An empirical investigation of the social coping strategies used by gifted adolescents. Gifted Child Quarterly, 39(3), 154-161.

Recebido em: 19/02/2015

Reformulado em: 28/06/2015

Aceito em: 13/07/2015

Sobre os autores:

Maria Luiza Pontes de França-Freitas é Doutora em Psicologia pelo Programa de Pós-Graduação em Psicologia da UFSCar, Mestre em Educação Especial pelo Programa de Pós-Graduação em Educação Especial da UFSCar. Membro do GT-Relações Interpessoaois e Competência Social da Associação Nacional de Pesquisa e Pós-Graduação em Psicologia (ANPEPP) desde 2010. Atualmente é Psicóloga Escolar Educacional da UFRN e desenvolve atividades voltadas para o ensino, pesquisa e extensão.

E-mail: mluizapf@gmail.com

Almir Del Prette é Doutor pela Psicologia, professor titular vinculado ao Departamento de Psicologia e aos Programas de Pós-Graduação em Psicologia e em Educação Especial da UFSCar, bolsista de produtividade em pesquisa do CNPq (Pq-1B), coordenadora do Grupo Relações Interpessoais e Habilidades Sociais (http://ww.rihs.ufscar.br), publicou em coautoria com Zilda Del Prette, vários livros, inventários e artigos sobre habilidades sociais.

E-mail:adprette@ufscar.br

Zilda A. P. Del Prette é Doutora em Psicologia, com pós-doutorado no exterior em Habilidades Sociais, professora titular sênior vinculada ao Departamento de Psicologia, ao Programa de Pós-Graduação em Psicologia da UFSCar e ao Instituto Nacional de Ciência e Tecnologia Comportamento, Cognição e Ensino (INCT/ECCE), Bolsista de Produtividade em Pesquisa do CNPq (1A), coordenadora do Grupo Relações Interpessoais e Habilidades Sociais (http://ww.rihs.ufscar.br), publicou, em coautoria com Almir Del Prette, artigos, livros e inventários de avaliação em Habilidades Sociais.

E-mail:zdprette@ufscar.br

Contato com os autores:

Maria Luiza Pontes de França-Freitas

Universidade Federal do Rio Grande do Norte, Escola de Ciências e Tecnologia.

AC Universidade Federal do Rio Grande do Norte

Bairro Lagoa Nova

Natal-RN, Brasil

CEP: 59078970 